\title{
Comparing the Gonadotoxicity of Multiple Breast Cancer Regimens: Important Understanding for Managing Breast Cancer in Pre-Menopausal Women
}

\author{
Valentino Martelli,2 \\ Maria Maddalena Latocca ${ }^{1,2}$ \\ Tommaso Ruelle ${ }^{1,2}$ \\ Marta Perachino ${ }^{1,2}$ \\ Luca Arecco ${ }^{1,2}$ \\ Kristi Beshiri,2 \\ Maria Grazia Razeti ${ }^{1,2}$ \\ Marco Tagliamento (D) 1,2 \\ Maurizio Cosso ${ }^{3}$ \\ Piero Fregatti (D) 4,5 \\ Matteo Lambertini (iD) ${ }^{1,2}$ \\ 'Department of Medical Oncology, U.O.C \\ Clinica Di Oncologia Medica, IRCCS \\ Ospedale Policlinico San Martino, Genova, \\ Italy; ${ }^{2}$ Department of Internal Medicine and \\ Medical Specialties (DiMI), School of \\ Medicine, University of Genova, Genova, \\ Italy; ${ }^{3}$ Department of Radiology, IRCCS \\ Ospedale Policlinico San Martino, Genova, \\ Italy; ${ }^{4}$ U.O.C. Clinica Di Chirurgia \\ Senologica, Department of Surgery, IRCCS \\ Ospedale Policlinico San Martino, Genova, \\ Italy; ${ }^{5}$ Department of Integrated Diagnostic \\ Surgical Sciences, School of Medicine, \\ University of Genova, Genova, Italy
}

\begin{abstract}
Over the last several decades, improvements in breast cancer treatment have contributed to increased cure rates for women diagnosed with this malignancy. Consequently, great importance should be paid to the long-term side effects of systemic therapies. For young women (defined as per guideline $\leq 40$ years at diagnosis) who undergo chemotherapy, one of the most impactful side effects on their quality of life is premature ovarian insufficiency (POI) leading to fertility-related problems and the side effects of early menopause. Regimens, type, and doses of chemotherapy, as well as the age of patients and their ovarian reserve at the time of treatment are major risk factors for treatment-induced POI. For these reasons, childbearing desire and preservation of ovarian function and/or fertility should be discussed with all premenopausal patients before planning the treatments. This manuscript summarizes the available fertility preservation techniques in breast cancer patients, the risk of treatment-induced POI with different anticancer treatments, and the possible procedures to prevent it. A special focus is paid to the role of oncofertility counseling, as a central part of the visit in this setting, during which the patient should receive all the information about the potential consequences of the disease and of the proposed treatment on her future life.
\end{abstract}

Keywords: breast cancer, fertility, young patient, oncofertility, premature ovarian insufficiency, anti-Mullerian hormone

\section{Introduction}

Breast cancer is the most common female malignancy worldwide: in 2020, there were 2.261.419 new diagnoses and about 680.000 women who died of this disease. ${ }^{1}$ A minority of cases affect the young population (defined as per guideline $\leq 40$ years at diagnosis), ${ }^{2}$ corresponding to less than $7 \%$ of all newly diagnosed tumors in Western Countries. $^{3,4}$ A higher proportion of cases in young patients are reported in less developed countries. ${ }^{5}$

When the diagnosis is prompt and the tumor is detected at an early stage, breast cancer is a curable disease through optimal integration of local and systemic therapies. ${ }^{6}$ The current standard chemotherapy for early breast cancer includes anthracycline- and taxane-based regimens. ${ }^{7}$ For specific subgroups of patients, these regimens are implemented with other compounds. Platinum salts combined with neoadjuvant anthracycline- and taxane-based regimens improve the rate of pathological complete response in triple-negative breast cancer. ${ }^{8}$ This disease subtype could also
Correspondence: Matteo Lambertini IRCCS Ospedale Policlinico San Martino, University of Genova, Largo Rosanna Benzi 10, Genova, 16132, Italy

Tel +39010 5554254

$\mathrm{Fax}+390105556536$

Email matteo.lambertini@unige.it 
benefit from immunotherapy, according to recent data. ${ }^{9}$ In HER2-positive disease, targeted therapy in addition to chemotherapy represents the standard of care. ${ }^{10,11}$ For hormonereceptor-positive early breast cancer, a crucial component of the treatment is represented by adjuvant endocrine therapy for 5-10 years. ${ }^{12}$ Three adjuvant endocrine therapy options are now available for premenopausal patients, according to their individual risk of recurrence: tamoxifen alone, the combination of ovarian suppression to tamoxifen, or to an aromatase inhibitor. ${ }^{13}$

In premenopausal women, systemic treatments may cause gonadal damage in several ways. This toxicity can lead to premature ovarian insufficiency (POI), fertility-related problems, and the side effects of early menopause. ${ }^{14,15}$ Therefore, in this subgroup of patients, oncofertility counseling is a central part of the medical consultation, since it allows the young patient to receive all the information about her risk of developing treatment-related POI, its potential impact on her quality of life, and the strategies available to reduce the burden of this side effect and to preserve fertility. Adequate counseling of the patients and answering all their questions at diagnosis are crucial steps to improve their quality of life before, during, and after active anticancer treatments. ${ }^{16}$

Systemic anticancer therapies and their dose as well as the age of patients and their ovarian reserve at the time of treatment are recognized risk factors for POI. Even though POI rates and the mechanisms of gonadotoxicity are relatively known for routinely used chemotherapy drugs, very poor data are now available about the gonadal effects of targeted therapies, immunotherapy, and endocrine therapy. This makes it difficult to perform appropriate oncofertility counseling in women candidates to receive these treatments. ${ }^{14,15}$
This manuscript summarizes the available fertility preservation techniques in breast cancer patients, the risk of treatment-induced POI with different anticancer treatments, and the possible procedures to prevent it. A special focus is paid to the role of oncofertility counseling, as a central part of the visit in this setting.

\section{Oncofertility Counselling}

Survivorship starts at diagnosis and continues throughout the entire lifespan ${ }^{17}$ during which patients can experience the long-term side effects of previous anticancer treatments. For young women, treatment-related POI represents an important concern and should be discussed during the first medical consultation. ${ }^{18}$

All the international guidelines strongly recommend the oncofertility counseling at diagnosis with all women diagnosed during their reproductive years, irrespective of their stage at diagnosis, including among women with advanced breast cancer. ${ }^{14,18,19}$ This counseling aims to clarify all the possible gonadal effects including POI and infertility as well as to clearly discuss all the available strategies to prevent them. ${ }^{20}$ In terms of timing, the sooner the patient receives the proper information, the earlier the access to fertility units can be planned for interested patients.

During the counseling, the first step is to assess the patient's childbearing desire. For young women wishing to have a future family, several fertility preservation strategies can be proposed, being oocyte (or embryo) cryopreservation the first to be discussed. ${ }^{21,22}$ The main features of these strategies are summarized in Table 1. Importantly, at a later time if these strategies are not successful or were not pursued at diagnosis, other

Table I Strategies for Preservation of Fertility and/or Ovarian Function in Women with Breast Cancer Diagnosed During Their Reproductive Years

\begin{tabular}{|l|l|l|l|l|}
\hline & $\begin{array}{l}\text { Time Needed } \\
\text { Before Systemic } \\
\text { Treatments }\end{array}$ & $\begin{array}{l}\text { Recommended } \\
\text { Age }\end{array}$ & Birth Rate & Contraindications \\
\hline $\begin{array}{l}\text { Oocyte/embryo } \\
\text { cryopreservation }\end{array}$ & $>14$ days & $\begin{array}{l}\text { Preferably } \leq 40 \\
\text { years }\end{array}$ & $43-62 \%^{* 32}$ & $\begin{array}{l}\text { Coagulation deficits; high risk of infection; non-compliance } \\
\text { with the required treatments and monitoring; urgent need } \\
\text { to start anticancer therapies }\end{array}$ \\
\hline $\begin{array}{l}\text { Ovarian tissue } \\
\text { cryopreservation }^{33}\end{array}$ & $2-3$ days & $\begin{array}{l}\text { Preferably } \leq 36 \\
\text { years }\end{array}$ & $\begin{array}{l}\text { About } \\
40 \%{ }^{34,35}\end{array}$ & $\begin{array}{l}\text { High surgical or anesthesiologic risks; high risk of ovarian } \\
\text { malignant contamination }\end{array}$ \\
\hline $\begin{array}{l}\text { Medical } \\
\text { gonadoprotection }\end{array}$ & $\sim 7$ days & $\begin{array}{l}\text { Premenopausal, } \\
\text { any age }\end{array}$ & $* *$ & Non-compliance with the treatment \\
\hline
\end{tabular}

Notes: *Birth rates of oocyte/embryo cryopreservation vary according to the patient's age and the number of oocytes collected. **This strategy has been studied as a technique to preserve ovarian function and not as a method for fertility preservation; nevertheless, an increased chance of pregnancy has been described. 
approaches like oocyte donation or adoption can be considered. $^{14}$

For premenopausal women older than 40 years who are not usually candidates for fertility preservation strategies as well as for all premenopausal women not interested in future childbearing, preservation of ovarian function should also be discussed to avoid the side effects of treatment-induced POI. ${ }^{23}$ Thus, infertility is not the only subject of oncofertility counseling, and the specialists have to give complete information also about the risks of POI and all its negative consequences, such as osteoporosis, higher risk of bone fractures, vaginal dryness, sexual dysfunction, hot flashes, and weight gain. ${ }^{14,18}$

Several studies have shown the importance of discussing ovarian function and fertility preservation in young breast cancer patients and their request to have access to the available strategies for reducing the burden of these side effects. ${ }^{24-26}$

Expert counseling of reproductive issues and modalities to reduce the potential consequences of POI is associated with higher consciousness about the disease, greater quality of life, and less regret about the decisions taken by the patients. ${ }^{16}$ Nevertheless, about $50 \%$ of all the patients do not receive the proper information about POI risk linked to the proposed anticancer treatments. ${ }^{27,28}$

Health care providers face several challenges during oncofertility counseling. ${ }^{25,29}$ For most doctors, the first issue is the lack of familiarity with fertility preservation strategies, due to inadequate knowledge on all the required steps during oncofertility counseling, their costs, and the involved technologies. However, as recommended by guidelines, ${ }^{14,18}$ this topic should be part of the education of all Medical Oncologists.

\section{Gonadotoxicity of Breast Cancer Treatments - Patient's Characteristics}

In addition to the proposed therapy, the gonadotoxicity of anticancer treatments in young women with breast cancer strongly depends also on patient-related characteristics (Figure 1). The main individual features of importance are age at the time of treatment, pre-treatment ovarian reserve, and the presence or not of pathogenic variants in the $B R C A$ genes. $^{14,15}$

\section{Age at the Time of Treatment}

This is a well-known crucial factor to estimate the risk of treatment-induced gonadotoxicity. ${ }^{37,38}$ The younger the patient, the lower the risk of developing POI with the same treatment due to the larger primordial follicle stockpile. So, the same regimen may have a high risk $(>80 \%)$ of post-treatment amenorrhea in women older than 40 years, while it may have a very low risk $(<20 \%)$ in women younger than 30 years at the beginning of therapy. Previous studies also suggest a longer period of menstrual function recovery in older women $(\geq 40$ years). ${ }^{39}$

\section{Pre-Treatment Ovarian Reserve}

Ovarian reserve has been traditionally defined as the woman's reproductive potential, assessed through the

\section{ASSESSING GONADOTOXICITY RISK}
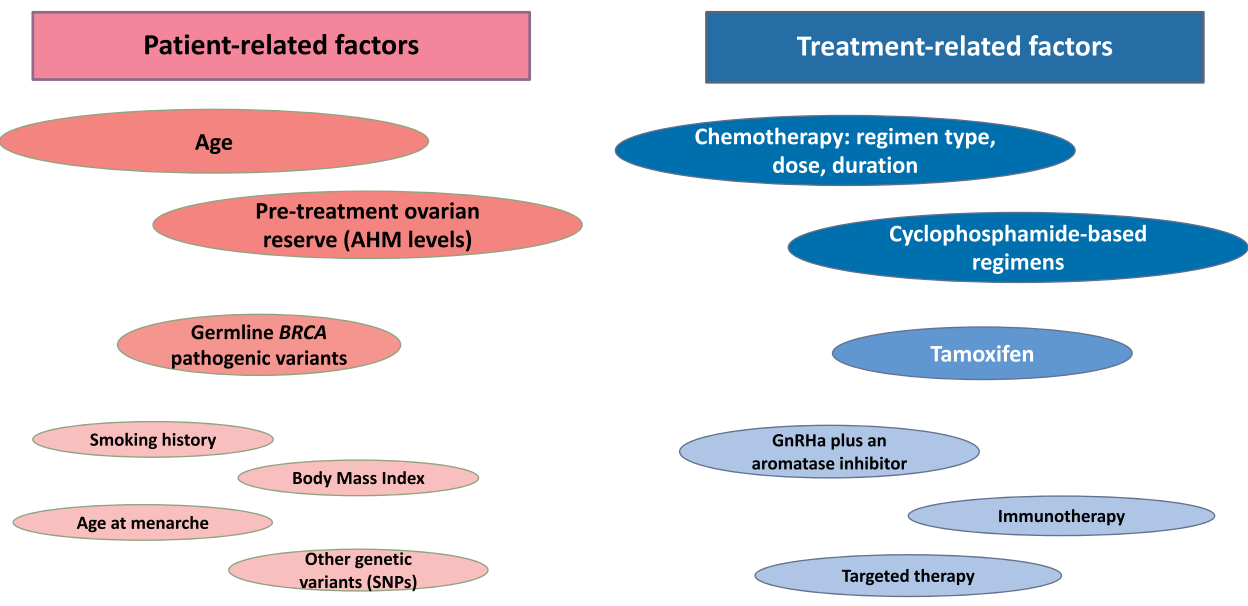

Figure I The most important factors affecting the gonadotoxicity risk in women with breast cancer patients receiving chemotherapy. Abbreviations: $\mathrm{AMH}$, anti-Mullerian hormone; $\mathrm{GnRH}$, gonadotropin-releasing hormone analog. 
quality and quantity of oocytes. ${ }^{40}$ It is influenced by age, genetics, and environmental factors and can be estimated in different ways. ${ }^{41}$ One of the easiest methods to perform it is the evaluation of anti-Mullerian hormone (AMH) serum levels, which is implied in the follicle recruitment and is considered an important marker of ovarian reserve. ${ }^{42}$

$\mathrm{AMH}$ levels help to assess the baseline ovarian reserve and to predict the risk of treatment-induced POI. ${ }^{43,44}$ Indeed, it decreases promptly after the start of chemotherapy and remains often undetectable for the whole period of treatment. Moreover, in several cases, AMH concentration continues to be undetectable even after the end of highly gonadotoxic systemic chemotherapy. ${ }^{44}$ Variations in the serum hormone levels through systemic treatments have been found to be similar in women with low, normal, or high levels at baseline. ${ }^{45}$ Note, however, that faster recovery of normal menstrual cyclicity has been observed in patients with high $\mathrm{AMH}$ concentration at the beginning and the end of therapies. ${ }^{46}$

\section{Germline Pathogenic Variants in Breast Cancer Susceptibility Genes}

Hereditary factors, in particular germline pathogenic variants in the $B R C A$ genes, are other important features that may potentially influence the risk of treatment-induced POI. ${ }^{4-49}$ Pathogenic variants of $B R C A$ lead to impaired DNA double-strand breaks repair mechanism; this can be associated with the decreased possibility to counteract genotoxic stress and the subsequent potential accelerated loss of ovarian reserve, following the accumulation of double-strand breaks in the oocytes. ${ }^{50}$

Evidence suggests that baseline ovarian reserve and performance of fertility preservation strategies may be impaired in breast cancer patients with germline pathogenic variants in the $B R C A$ genes. ${ }^{51}$ Limited evidence is available to counsel these patients on their potential higher risk of treatment-induced POI, with two studies that did not show differences in amenorrhea rates ${ }^{52}$ or AMH levels ${ }^{53}$ following chemotherapy completion between breast cancer patients with or without germline pathogenic variants in the $B R C A$ genes. Only one study suggested a potential increased reduction in post-treatment AMH levels for BRCA-mutated patients. $^{55}$ This topic remains a burning question in the oncofertility counseling of young breast cancer patients and future research efforts are needed. ${ }^{14,15}$

\section{Other Patients' Characteristics}

The impact of other anthropometric and lifestyle factors and the potential role of additional genetic factors on the risk of treatment-induced gonadotoxicity remains to be fully clarified.

Abusief et al analyzed the relationship between chemotherapy-induced amenorrhea and patients' characteristics in a retrospective cohort of young women with breast cancer. ${ }^{55}$ Results were not conclusive for all the analyzed features; it is interesting to note that body mass index did not affect the chance of amenorrhea; on the contrary the menarche after the age of 13 years was associated with a higher probability of amenorrhea. Also, cigarette smokers could increase the risk, but this relation remains not completely clear. ${ }^{55}$

Genetic variants (eg single nucleotide polymorphisms, SNPs) have been studied for their potential role in influencing the risk of POI. ${ }^{56}$ However, also, in this case, further studies are needed to confirm their role in influencing the risk of treatment-induced POI.

\section{Gonadotoxicity of Breast Cancer Treatments - Chemotherapy}

Standard oncological therapies for breast cancer can lead to POI through three main different coexisting mechanisms: by directly damaging ovarian follicular cells (both growing and non-growing follicles), by accelerating follicular activation depleting primordial pool via increased activation, ${ }^{57}$ and by damaging the ovarian stroma also by altering ovarian blood supply. ${ }^{58}$ The majority of the chemotherapy compounds used in routine clinical practice can induce these damages: the type of therapy, its dose, and the duration are crucial factors (Figure 1).

\section{Cyclophosphamide}

In early breast cancer, one of the key agents used as (neo) adjuvant treatment with the highest risk of gonadotoxicity is the alkylating agent cyclophosphamide. Regimens including cyclophosphamide have a significantly higher risk of POI compared to those not including it. Indeed, these agents are associated with a possible damage to nongrowing primordial follicle pool which makes up the ovarian reserve. Patients treated with such treatment have more than double chances of developing treatment-induced amenorrhea (OR 2.25; 95\% CI 1.26-4.03, P =0.006). ${ }^{59}$

Significant risk variations are observed according to patient's age, dose of cyclophosphamide, regimen, and chosen schedule. More precisely, the highest risk of 
amenorrhea ( $>80 \%$ ) is linked to cyclophosphamide-based regimens like $\mathrm{CMF}, \mathrm{CEF}, \mathrm{CAF}$, or TAC for 6 cycles in women older than 40 years; women between 30 and 39 years treated with the same regimens have an intermediate risk of amenorrhea (40-60\%), like those older than 40 years treated with $\mathrm{AC}$ or $\mathrm{EC}$ for 4 cycles. Finally, a lower risk $(<20 \%)$ exists for CMF, CEF, CAF, TAC for 6 cycles in patients younger than 30 years and for $\mathrm{AC}$ for 4 cycles in women younger than 40 years. ${ }^{60}$

\section{Anthracyclines and Taxanes}

Anthracyclines and taxanes are two other cornerstones in the (neo)adjuvant treatment of breast cancer. The former is associated with a significant increase in the risk of chemotherapy-induced amenorrhea (OR 1.39; 95\% CI $1.15-1.70, \mathrm{P}=0.0008$ ); a similar trend has been also observed for the latter (OR 1.24; 95\% CI 1.03-1.50, $\mathrm{P}=0.02) .{ }^{59} \mathrm{AMH}$ levels drop rapidly to undetectable levels in the majority of cases after their administration and generally remain very low even during the follow-up period. ${ }^{44}$ In particular, a long-lasting impact of these compounds on AMH has been observed up to 3 years after diagnosis and treatment. ${ }^{53}$

To date, in routine clinical practice, (neo)adjuvant chemotherapy consists of sequential treatment with anthracycline plus cyclophosphamide followed by a taxane or the combination of cyclophosphamide plus docetaxel (TC regimen). Women treated with taxanes have an higher rate of menopause than those not exposed to this agent, as observed by Silva et al (OR 0.49; 95\% CI, 0.30-0.80; $\mathrm{P}=0.004)^{38}$ and Lambertini et al (OR 1.92, 95\% CI, 1.44-2.56; $\mathrm{P}<0.001) .{ }^{62}$ Indeed, taxanes following anthracycline plus cyclophosphamide regimens significantly reduce serum AMH levels one year after treatment completion $(0.22$ vs $0.04 \mu \mathrm{g} / \mathrm{L}, \mathrm{P}=0.0006) .{ }^{53}$ Similar rates of treatment-induced amenorrhea are expected with the combination of cyclophosphamide plus a taxane or the sequential administration of an anthracycline plus cyclophosphamide followed by a taxane $(81 \%$ and $80 \%$ of patients reported cessation of menses after chemotherapy). ${ }^{62}$

In routine clinical practice, high-risk patients with early breast cancer may receive a dose-dense schedule (ie similar dose as a standard treatment but with a shorter interval between the cycles). This approach does not seem to increase the risk of developing treatmentinduced amenorrhea as compared to the same chemotherapy regimen given with a standard schedule (OR 1.00; 95\% CI 0.80-1.25, $\mathrm{P}=0.989) .{ }^{63}$

\section{Anti-HER2 Agents}

Although the use of anti-HER2 therapies has significantly increased in the past few years, with numerous drugs and several indications in different settings, to date, there is limited evidence on their risk of gonadotoxicity and fertility impairment. HER2-directed agents do not seem to have significant ovarian toxicity. ${ }^{64}$ In particular, the use of trastuzumab does not seem to increase the risk of treatment-related amenorrhea. ${ }^{65}$

An unplanned analysis of the ALTTO trial was conducted to evaluate the rate of treatment-related amenorrhea with anti-HER2 therapies. The study population was composed of women with HER2 positive early disease randomly assigned (1:1:1:1) to receive chemotherapy and anti-HER2 therapy with trastuzumab alone, trastuzumab followed by lapatinib, trastuzumab and lapatinib, or lapatinib alone as adjuvant therapy. The incidence of amenorrhea was $72.6 \%$ for trastuzumab monotherapy, $72.1 \%$ for the sequential approach, $74.8 \%$ in the combination arm, and $74.0 \%$ for the lapatinib monotherapy $(\mathrm{p}=0.64)$. The trial design did not include a control arm without anti-HER2 therapy, so it is hard to assess the real effects of these drugs on the risk of post-treatment amenorrhea considering that all patients were also first exposed to chemotherapy. However, a higher incidence of treatment-related amenorrhea was not observed in the dual-blockade arms compared to monotherapy ones. This may suggest the gonadal safety of these anti-HER2 agents. ${ }^{61}$

The phase II, single-arm APT trial evaluated the efficacy of paclitaxel combined with trastuzumab for 12 weeks followed by 36 weeks of anti-HER2 antibody monotherapy. ${ }^{66}$ Ruddy et al analyzed its data, focusing on the rate of chemotherapy-related amenorrhea ${ }^{67}$ Of the 410 patients enrolled, 64 women were in a premenopausal status and eligible for the analysis. These had to answer to menstrual survey every 12-48 weeks for 6 years; median age was 44 and the median duration of the survey was 51 months (time between first cycle and last survey). Most of the patients recovered their normal menstrual cyclicity at the time of the last survey $(72 \%, 95 \%$ CI $59-82 \%)$, showing a lower incidence of POI with adjuvant paclitaxel and trastuzumab than with anthracyclines-taxanes regimens.

Pertuzumab is another intravenous anti-HER2 antibody, used with trastuzumab also in early settings as both neoadjuvant and adjuvant therapy. ${ }^{68,69}$ There is a lack of evidence about its possible effects on fertility. ${ }^{14}$ 
Neratinib is an anti-HER 2 drug taken orally and used in an adjuvant setting after trastuzumab-based therapy. ${ }^{70}$ Considering the lack of data in this regard, it is hard to assess the real impact of this compound on fertility. Amenorrhea rates in premenopausal patients that had received this treatment were not reported in the registration trial. ${ }^{71}$

T-DM1 is an antibody-drug conjugate, which combines a targeted therapy (ie trastuzumab) with a cytotoxic agent (ie DM1, a derivative of maytansine, targeting tubulin). ${ }^{72}$ This agent proved to be highly effective also in the early setting, for patients without pathologic complete response following trastuzumab-based therapy. ${ }^{73}$ Some data on the potential gonadotoxicity of T-DM1 are derived from the analysis by Ruddy et al within the ATEMPT trial, a study that compared paclitaxel plus trastuzumab versus T-DM1 as an adjuvant therapy for stage I HER2-positive disease. ${ }^{74}$ All the enrolled premenopausal subjects had to complete a menstrual survey at baseline and every 6-12 months, during the 3-year follow up. At 18 months, less than $50 \%$ of patients in the control arm had menses in the previous 6 months compared to $75 \%$ of women treated with T-DM1 $(p=0.011)$. All the patients in the experimental arm $\leq 40$ years reported menstruation at 1.5-year follow-up. Although these data suggest a potential gonadal safety profile for T-DM1, future studies are needed for a better understanding of its potential gonadotoxicity, especially in women previously exposed to chemotherapy as currently recommended in early settings.

\section{CDK4/6 Inhibitors}

This category comprehends palbociclib, ribociclib, and abemaciclib, largely used in metastatic hormone receptorpositive/HER2-negative disease. Recent data have shown the potential efficacy of abemaciclib as adjuvant therapy, for two years in addition to endocrine therapy in high-risk patients with hormone receptor-positive/HER2-negative early breast cancer. ${ }^{75}$ Despite $43.5 \%$ of patients included in this trial were premenopausal, no data have been reported so far on the incidence of post-treatment amenorrhea (and the follow-up of the trial is too short to assess this outcome). While palbociclib has not shown a beneficial effect in the early setting, ${ }^{76}$ results of the adjuvant ribociclib trial are awaited.

\section{PARP Inhibitors}

The pharmacological category of poly (ADP-ribose) polymerase (PARP) inhibitors is composed of two small molecules used in BRCA-mutated breast cancer patients with advanced disease: olaparib and talazoparib. ${ }^{77}$ Results of the adjuvant olaparib trial in early-stage breast cancer are awaited to potentially open the door to also use this agent as adjuvant therapy following standard chemotherapy.

The only existing data on the gonadotoxicity of PARP inhibitors have been provided in mice. The study conducted by Winship and colleagues evaluated the influence of classical anticancer agents (ie cyclophosphamide, doxorubicin, carboplatin, or paclitaxel) administered alone or with olaparib in $B R C A$ wild-type female mice. Primordial follicles were the most affected cellular lines by the combination arm with a dramatic depletion of $36 \%$ versus control arm (standard anticancer agents, without PARP inhibitor $)(\mathrm{p}<0.05)$. Other follicle cells, ovulation, and $\mathrm{AMH}$ levels showed no consequences. It is interesting to note that, if combined with other agents, olaparib does not seem to exacerbate chemotherapy-induced gonadal damage. $^{78}$

These results represent a first step in the comprehension of the impact of PARP inhibitors on fertility; however, further preclinical and clinical studies are required.

\section{Immune Checkpoint Inhibitors}

Immune checkpoint inhibitors (ICIs) have revolutionized the treatment landscape of different tumors. ${ }^{79,80}$

Recently, different trials have focused on the efficacy of ICIs in early breast cancer patients with triple-negative disease. Results from Keynote-522 and IMpassion031 showed the effect of adding an ICI (pembrolizumab and atezolizumab, respectively) to neoadjuvant chemotherapy in increasing the rate of pathologic complete responses. ${ }^{9}$ In these studies, reproductive side effects have not been described. The only indirect link to menopausal status is "hot flushes" in IMpassion031: there were 15\% any grade hot flushes in the atezolizumab plus chemotherapy arm and $10 \%$ in the placebo plus chemotherapy arm, without grade $3-4$ events. $^{9}$

Assessing how fertility can be impaired by immunotherapy is fundamental since these drugs will be used in early settings for many young patients with different diseases $^{81}$ and ICIs can influence almost all the endocrine pathways, including reproductive ones. ${ }^{82}$

For this reason, the National Comprehensive Cancer Network guidelines suggest adopting birth control methods during and for at least 5 months after the end of immunotherapy. ${ }^{83}$

Given the lack of knowledge in this field, further studies are needed to establish the real incidence of 
gonadotoxicity and fertility impairment in breast cancer patients treated with immunotherapy.

\section{Endocrine Therapy}

Regarding endocrine therapy, the use of tamoxifen does not appear to hurt the ovarian reserve with several studies showing no significant differences in AMH levels between patients receiving or not this treatment after chemotherapy. ${ }^{43,44,53}$ However, tamoxifen can cause alterations in the menstrual cycles by significantly increasing the risk of developing amenorrhea following chemotherapy completion (OR 1.48; 95\% CI 1.28-1.70, P < 0.0001). ${ }^{59}$ However, since tamoxifen stimulates the ovaries, it can cause gonadal alterations.

The use of gonadotropin-releasing hormone analogs (GnRHa) causes a suspension of the normal menstrual cycle which is reversible in $90 \%$ of cases in patients younger than 40 years; menstrual function recovery is not always observed in older patients. ${ }^{84}$

The risk of POI with the administration of GnRH analogs associated with an aromatase inhibitor has not yet been investigated.

\section{Gonadotropin-Releasing Hormone Agonists as a Strategy to Reduce Chemotherapy-Induced Gonadotoxicity}

Pharmacological ovarian suppression with GnRHa concomitantly with chemotherapy has been studied for years as a way to reduce the risk of POI. ${ }^{23}$ Currently, this is the only medical intervention with proven efficacy in reducing the risk of chemotherapy-induced gonadotoxicity. ${ }^{14,15}$

It represents the gold standard in women willing to avoid chemotherapy-induced POI, irrespective of their future desire for pregnancy. Some of its advantages are its non-invasiveness and accessibility: it should be started at least one week before the beginning of chemotherapy and should be continued during all the period of cytotoxic therapy. ${ }^{23}$

This strategy seems not only to protect from the risk of POI but also the degree of recovery of the ovarian reserve. In a prospective study by Sinha et al antral follicle count (AFC) has been assessed before and after chemotherapy for breast cancer: in those patients who underwent GnRHa, the AFC recovery was faster and to a higher rate compared to those that did not receive GnRHa. ${ }^{85}$
In the last two decades, several randomized trials evaluated the efficacy of GnRHa use in reducing the risk of POI when administered during chemotherapy in breast cancer patients. ${ }^{23}$ Among these studies, the largest have clearly shown that the administration of GnRHa during chemotherapy significantly reduces the risk of chemotherapy-induced POI. ${ }^{86-88}$

The meta-analysis by Lambertini et al confirmed the efficacy and the safety of this treatment, merging individual patient-level data from the five major trials (PROMISE-GIM6; POEMS; OPTION; GBG-37; ZORO). The overall population was composed of 872 premenopausal breast cancer patients, randomly assigned to receive GnRHa (436 women) or not (control group, 437 women) during chemotherapy. ${ }^{36}$ The experimental arm showed POI in $14 \%$ of cases, whereas in the control group the incidence was higher $(30.9 \%)(\mathrm{p}<0.001)$. Concerning pregnancies after chemotherapy, more favorable data have been found in the treated group: $10 \%$ of women completed at least a gestation, compared to $5.5 \%$ in the control $(\mathrm{p}=0.03)$. In terms of safety, similar DFS and OS were found in the two groups, irrespective of hormone receptor status.

These results are in line with those of a previous metaanalysis, performed in 2015 with abstracted data collected from 12 trials, including 1231 early breast cancer patients undergoing chemotherapy with or without GnRHa. ${ }^{89}$ Overall, POI was higher for patients treated with chemotherapy alone $(33.5 \%$ versus $18.5 \%$, respectively; $\mathrm{p}<$ $0.001)$. The pregnancy rate was more favorable in patients treated with chemotherapy plus GnRHa $(9.2 \%)$ than in those who received cytotoxic therapy alone $(5.5 \%)$.

One of the concerns about the administration of GnRHa during chemotherapy was the fear that GnRHa could antagonize cytotoxic therapy in patients with hormone receptor-positive disease ${ }^{90}$ However, no difference in DFS and OS was observed in patients with hormone receptor-positive disease who received or did not receive GnRHa concomitantly with chemotherapy. ${ }^{89,91}$ Furthermore, two recent studies (TEXT and SOFT studies) investigating different adjuvant endocrine therapy approaches showed no difference in DFS and OS in women receiving GnRHa concomitantly or sequentially to chemotherapy. ${ }^{92}$

Over the past years, the use of GnRHa has been highly debated being the lack of evidence about its mechanism of protective effect the main reason to criticize its use. ${ }^{93}$

However, given all these efficacy and safety data, nowadays international guidelines recommend GnRHa 
administration as a valid strategy to preserve ovarian function (but not an alternative to cryopreservation strategies for fertility preservation) in premenopausal breast cancer patients that undergo chemotherapy. ${ }^{14,15,18}$

\section{Conclusions}

With an increased lifespan, many young breast cancer survivors may face the long-term consequences of the side effects of anticancer therapies, including the risk of gonadal damage and fertility impairment.

In the past decades, more attention has been paid to oncofertility and nowadays there is a growing evidence about the risk of POI with the different treatments, the chances of pregnancy after treatment, and the strategies to preserve ovarian function and fertility. However, there are controversial data and sometimes a lack of evidence on the potential effect on patients' ovarian function and fertility of many new effective anticancer therapies that have entered clinical use in the curative setting or are in latestage of clinical development. This raises the need to pursue additional research efforts in this area. ${ }^{94}$

With oncofertility counseling being a central part of the first medical consultation, providing a proper estimation of the risk of treatment-induced gonadotoxicity represents a key step to allow young women to make informed decisions on the proposed anticancer therapies as well as on their wish to access the available strategies for ovarian function and/or fertility preservation.

\section{Acknowledgments}

Matteo Lambertini acknowledges the Italian Ministry of Health $-5 \times 1000$ funds 2017 (no grant number) and the Italian Association for Cancer Research (AIRC; MFAG 2020 ID 24698) for pursuing his research efforts in the field of oncofertility.

\section{Disclosure}

Matteo Lambertini acted as consultant for Roche, AstraZeneca, Lilly and Novartis, and received speaker honoraria from Sandoz, Roche, Takeda, Pfizer, Lilly and Novartis outside the submitted work. Marco Tagliamento reports travel and accommodation expenses supported by Roche, Bristol-Myers Squibb, AstraZeneca and Takeda; and activity as a medical writer supported by Novartis and Amgen, outside the submitted work. All the other authors declare no conflicts of interest.

\section{References}

1. Sung H, Ferlay J, Siegel RL, et al. Global cancer statistics 2020: GLOBOCAN estimates of incidence and mortality worldwide for 36 cancers in 185 countries. CA Cancer J Clin. 2021:41.

2. Paluch-Shimon S, Cardoso F, Partridge AH, et al. ESO-ESMO 4th International Consensus Guidelines for Breast Cancer in Young Women (BCY4). Ann Oncol. 2020;31(6):674-696. doi:10.1016/j. annonc.2020.03.284

3. Fidler MM, Gupta S, Soerjomataram I, Ferlay J, Steliarova-Foucher E, Bray F. Cancer incidence and mortality among young adults aged 20-39 years worldwide in 2012: a population-based study. Lancet Oncol. 2017;18(12):1579-1589. doi:10.1016/S1470-2045(17)30677-0

4. Siegel RL, Miller KD, Jemal A. Cancer statistics, 2020. CA Cancer J Clin. 2020;70(1):7-30. doi:10.3322/caac.21590

5. Ghiasvand R, Adami H-O, Harirchi I, Akrami R, Zendehdel K. Higher incidence of premenopausal breast cancer in less developed countries; myth or truth? BMC Cancer. 2014;14(1):343. doi:10.1186/ 1471-2407-14-343

6. Harbeck N, Gnant M. Breast cancer. The Lancet. 2017;389 (10074):1134-1150. doi:10.1016/S0140-6736(16)31891-8

7. Caparica R, Bruzzone M, Poggio F, et al. Anthracycline and taxanebased chemotherapy versus docetaxel and cyclophosphamide in the adjuvant treatment of HER2-negative breast cancer patients: a systematic review and meta-analysis of randomized controlled trials. Breast Cancer Res Treat. 2019;174(1):27-37.

8. Schmid P, Cortes J, Pusztai L, et al. Pembrolizumab for Early TripleNegative Breast Cancer. N Engl J Med. 2020;382(9):810-821. doi:10.1056/NEJMoa1910549

9. Mittendorf EA, Zhang H, Barrios $\mathrm{CH}$, et al. Neoadjuvant atezolizumab in combination with sequential nab-paclitaxel and anthracycline-based chemotherapy versus placebo and chemotherapy in patients with early-stage triple-negative breast cancer (IMpassion031): a randomised, double-blind, phase 3 trial. The Lancet. 2020;396 (10257):1090-1100. doi:10.1016/S0140-6736(20)31953-X

10. Cardoso F, Kyriakides S, Ohno S, et al. Early breast cancer: ESMO Clinical Practice Guidelines for diagnosis, treatment and follow-up. Ann Oncol. 2019;30(8):1194-1220. doi:10.1093/annonc/mdz173

11. Korde LA, Somerfield MR, Carey LA, et al. Neoadjuvant Chemotherapy, Endocrine Therapy, and Targeted Therapy for Breast Cancer: ASCO Guideline. J Clin Oncol. 2021:JCO.20.03399. doi:10.1200/JCO.20.03399

12. Burstein HJ, Lacchetti C, Anderson $\mathrm{H}$, et al. Adjuvant Endocrine Therapy for Women With Hormone Receptor-Positive Breast Cancer: ASCO Clinical Practice Guideline Focused Update. J Clin Oncol. 2019;37(5):423-438. doi:10.1200/JCO.18.01160

13. Lambertini M, Blondeaux E, Perrone F, Del Mastro L. Improving adjuvant endocrine treatment tailoring in premenopausal women with hormone receptor-positive breast cancer. J Clin Oncol. 2020;38 (12):1258-1267. doi:10.1200/JCO.19.02242

14. Lambertini M, Peccatori FA, Demeestere I, et al. Fertility preservation and post-treatment pregnancies in post-pubertal cancer patients: ESMO Clinical Practice Guidelinest. Ann Oncol. 2020;31 (12):1664-1678. doi:10.1016/j.annonc.2020.09.006

15. Anderson RA, Amant F, Braat D; The ESHRE Guideline Group on Female Fertility Preservation. ESHRE guideline: female fertility preservation†. Hum Reprod Open. 2020;2020(4):hoaa052. doi:10.1093/ hropen/hoaa052

16. Letourneau JM, Ebbel EE, Katz PP, et al. Pretreatment fertility counseling and fertility preservation improve quality of life in reproductive age women with cancer. Cancer. 2012;118(6):1710-1717. doi:10.1002/cncr.26459

17. Franzoi MA, Agostinetto E, Perachino M, et al.Evidence-based approaches for the management of side-effects of adjuvant endocrine therapy in patients with breast cancer. Lancet Oncol. 2021. doi:10.1016/S1470-2045(20)30666-5. 
18. Oktay K, Harvey BE, Partridge AH, et al. Fertility Preservation in Patients With Cancer: ASCO Clinical Practice Guideline Update. $J$ Clin Oncol. 2018;36(19):1994-2001. doi:10.1200/JCO.20 18.78.1914

19. Cardoso F, Paluch-Shimon S, Senkus E, et al. 5th ESO-ESMO international consensus guidelines for advanced breast cancer (ABC 5). Ann Oncol. 2020;31(12):1623-1649. doi:10.1016/j.annonc.20 20.09.010

20. Lambertini M, Anserini P, Levaggi A, Poggio F, Mastro LD. Fertility counseling of young breast cancer patients. J Thorac Dis. 2013;5(1): S68-80. doi:10.3978/j.issn.2072-1439.2013.05.22

21. von Wolff M, Capp E, Jauckus J, Strowitzki T, Germeyer A. Timing of ovarian stimulation in patients prior to gonadotoxic therapy: an analysis of 684 stimulations. Eur J Obstet Gynecol Reprod Biol. 2016;199:146-149. doi:10.1016/j.ejogrb.2016.02.006

22. Rienzi L, Gracia C, Maggiulli R, et al. Oocyte, embryo and blastocyst cryopreservation in ART: systematic review and meta-analysis comparing slow-freezing versus vitrification to produce evidence for the development of global guidance. Hum Reprod Update. 2016. doi:10.1093/humupd/dmw038

23. Lambertini M, Horicks F, Del mastro L, Partridge AH, Demeestere I. Ovarian protection with gonadotropin-releasing hormone agonists during chemotherapy in cancer patients: from biological evidence to clinical application. Cancer Treat Rev. 2019;72:65-77. doi:10.1016/j. ctrv.2018.11.006

24. Ruddy KJ, Gelber SI, Tamimi RM, et al. Prospective Study of Fertility Concerns and Preservation Strategies in Young Women With Breast Cancer. $J$ Clin Oncol. 2014;32(11):1151-1156. doi:10.1200/JCO.2013.52.8877

25. Lambertini M, Di Maio M, Pagani O, et al. The BCY3/BCC 2017 survey on physicians' knowledge, attitudes and practice towards fertility and pregnancy-related issues in young breast cancer patients. The Breast. 2018;42:41-49. doi:10.1016/j.breast.2018.08.099

26. Ruggeri M, Pagan E, Bagnardi V, et al. Fertility concerns, preservation strategies and quality of life in young women with breast cancer: baseline results from an ongoing prospective cohort study in selected European Centers. The Breast. 2019;47:85-92. doi:10.1016/j. breast.2019.07.001

27. Lee RJ, Wakefield A, Foy S, Howell SJ, Wardley AM, Armstrong AC. Facilitating reproductive choices: the impact of health services on the experiences of young women with breast cancer. Psychooncology. 2011;20(10):1044-1052. doi:10.1002/ pon. 1826

28. Goossens J, Delbaere I, Van Lancker A, Beeckman D, Verhaeghe S, Van Hecke A. Cancer patients' and professional caregivers' needs, preferences and factors associated with receiving and providing fertility-related information: a mixed-methods systematic review. Int $J \quad$ Nurs Stud. 2014;51(2):300-319. doi:10.1016/j. ijnurstu.2013.06.015

29. Covelli A, Facey M, Kennedy E, et al. Clinicians' Perspectives on Barriers to Discussing Infertility and Fertility Preservation With Young Women With Cancer. JAMA Netw Open. 2019;2(11): e1914511. doi:10.1001/jamanetworkopen.2019.14511

30. Oktay K, Turan V, Bedoschi G, Pacheco FS, Moy F. Fertility Preservation Success Subsequent to Concurrent Aromatase Inhibitor Treatment and Ovarian Stimulation in Women With Breast Cancer. $J$ Clin Oncol off J Am Soc Clin Oncol. 2015;33(22):2424-2429. doi:10.1200/JCO.2014.59.3723

31. Diaz-Garcia C, Domingo J, Garcia-Velasco JA, et al. Oocyte vitrification versus ovarian cortex transplantation in fertility preservation for adult women undergoing gonadotoxic treatments: a prospective cohort study. Fertil Steril. 2018;109(3):478-485.e2. doi:10.1016/j. fertnstert.2017.11.018

32. Cobo A, García-Velasco J, Domingo J, Pellicer A, Remohí J. Elective and Onco-fertility preservation: factors related to IVF outcomes. Hum Reprod. 2018;33(12):2222-2231. doi:10.1093/humrep/dey321
33. Gellert SE, Pors SE, Kristensen SG, Bay-Bjørn AM, Ernst E, Yding Andersen C. Transplantation of frozen-thawed ovarian tissue: an update on worldwide activity published in peer-reviewed papers and on the Danish cohort. J Assist Reprod Genet. 2018;35 (4):561-570. doi:10.1007/s10815-018-1144-2

34. Meirow D, Ra'anani H, Shapira M, et al. Transplantations of frozen-thawed ovarian tissue demonstrate high reproductive performance and the need to revise restrictive criteria. Fertil Steril. 2016;106(2):467-474. doi:10.1016/j.fertnstert.2016.04.031

35. Pacheco F, Oktay K. Current Success and Efficiency of Autologous Ovarian Transplantation: a Meta-Analysis. Reprod Sci Thousand Oaks Calif. 2017;24(8):1111-1120. doi:10.1177/1933719117702251

36. Lambertini M, Moore HCF, Leonard RCF, et al. GonadotropinReleasing Hormone Agonists During Chemotherapy for Preservation of Ovarian Function and Fertility in Premenopausal Patients With Early Breast Cancer: a Systematic Review and MetaAnalysis of Individual Patient-Level Data. J Clin Oncol. 2018;36 (19):1981-1990. doi:10.1200/JCO.2018.78.0858

37. Su HI, Haunschild C, Chung K, et al. Pre-chemotherapy anti-mullerian hormone, age and body size predict timing of return of ovarian function in young breast cancer patients. Cancer. 2014;120 (23):3691-3698. doi:10.1002/cncr.28942

38. Silva C, Caramelo O, Almeida-Santos T, Ribeiro Rama AC. Factors associated with ovarian function recovery after chemotherapy for breast cancer: a systematic review and meta-analysis. Hum Reprod. 2016;31(12):2737-2749. doi:10.1093/humrep/dew224

39. Lee S, Kil WJ, Chun M, et al. Chemotherapy-related amenorrhea in premenopausal women with breast cancer: menopause. Menopause (New York, N.Y.). 2009;16(1):98-103. doi:10.1097/ gme.0b013e3181844877

40. Practice Committee of the American Society for Reproductive Medicine. Testing and interpreting measures of ovarian reserve: a committee opinion. Fertil Steril. 2015;103(3):e9-e17. doi:10.1016/j.fertnstert.2014.12.093

41. Tal R, Seifer DB. Ovarian reserve testing: a user's guide. Am J Obstet Gynecol. 2017;217(2):129-140. doi:10.1016/j.ajog.2017.02.027

42. Anderson RA, Cameron DA. Pretreatment Serum Anti-Müllerian Hormone Predicts Long-Term Ovarian Function and Bone Mass after Chemotherapy for Early Breast Cancer. J Clin Endocrinol Metab. 2011;96(5):1336-1343. doi:10.1210/jc.2010-2582

43. Anderson RA, Mansi J, Coleman RE, Adamson DJA, Leonard RCF. The utility of anti-Müllerian hormone in the diagnosis and prediction of loss of ovarian function following chemotherapy for early breast cancer. Eur J Cancer. 2017;87:58-64. doi:10.1016/j.ejca.2017.10.001

44. Fréour T, Barrière P, Masson D. Anti-müllerian hormone levels and evolution in women of reproductive age with breast cancer treated with chemotherapy. Eur J Cancer. 2017;74:1-8. doi:10.1016/j.ejca.2016.12.008

45. Loubersac S, Dezellus A, Lefebvre T, et al. Evolution of serum AntiMüllerian Hormone (AMH) level in young women treated with chemotherapy for breast cancer according to basal AMH level. Eur J Obstet Gynecol Reprod Biol. 2020;254:132-137. doi:10.1016/j. ejogrb.2020.09.016

46. Dillon KE, Sammel MD, Prewitt M, et al. Pre-treatment amh determines rate of post-therapy ovarian reserve recovery: acute changes in ovarian reserve during and after chemotherapy. Fertil Steril. 2013;99 (2):477-483. doi:10.1016/j.fertnstert.2012.09.039

47. Lambertini M, Goldrat O, Toss A, et al. Fertility and pregnancy issues in BRCA-mutated breast cancer patients. Cancer Treat Rev. 2017;59:61-70. doi:10.1016/j.ctrv.2017.07.001

48. Vuković P, Peccatori FA, Massarotti C, Miralles MS, BeketićOrešković L, Lambertini M. Preimplantation genetic testing for carriers of BRCA1/2 pathogenic variants. Crit Rev Oncol Hematol. 2021;157:103201. doi:10.1016/j.critrevonc.2020.103201

49. Turan V, Oktay K. BRCA-related ATM-mediated DNA double-strand break repair and ovarian aging. Hum Reprod Update. 2020;26 (1):43-57. doi:10.1093/humupd/dmz043 
50. Turan V, Lambertini M, Lee DY, et al. Association of Germline BRCA Pathogenic Variants With Diminished Ovarian Reserve: A Meta-Analysis of Individual Patient-Level Data. J Clin Oncol. 2021. doi:10.1200/JCO.20.02880

51. Lambertini M, Goldrat O, Ferreira AR, et al. Reproductive potential and performance of fertility preservation strategies in BRCA-mutated breast cancer patients. Ann Oncol. 2018;29(1):237-243. doi:10.1093/ annonc/mdx639

52. Valentini A, Finch A, Lubiński J, et al. Chemotherapy-Induced Amenorrhea in Patients With Breast Cancer With a BRCA1 or BRCA2 Mutation. J Clin Oncol. 2013;31(31):3914-3919. doi:10.1200/JCO.2012.47.7893

53. Lambertini $\mathrm{M}$, Olympios $\mathrm{N}$, Lequesne $\mathrm{J}$, et al. Impact of Taxanes, Endocrine Therapy, and Deleterious Germline BRCA Mutations on Anti-müllerian Hormone Levels in Early Breast Cancer Patients Treated With Anthracycline- and Cyclophosphamide-Based Chemotherapy. Front Oncol. 2019;9:575. doi:10.3389/fonc.2019.00575

54. Oktay KH, Bedoschi G, Goldfarb SB, et al. Increased chemotherapy-induced ovarian reserve loss in women with germline BRCA mutations due to oocyte deoxyribonucleic acid double strand break repair deficiency. Fertil Steril. 2020;113(6):1251-1260.e1. doi:10.1016/j.fertnstert.2020.01.033

55. Abusief ME, Missmer SA, Ginsburg ES, Weeks JC, Partridge AH. Relationship between reproductive history, anthropometrics, lifestyle factors, and the likelihood of persistent chemotherapy-related amenorrhea in women with premenopausal breast cancer. Fertil Steril. 2012;97(1):154-159. doi:10.1016/j.fertnstert.2011.10.005

56. Ruddy KJ, Schaid DJ, Partridge AH, et al. Genetic predictors of chemotherapy-related amenorrhea in women with breast cancer. Fertil Steril. 2019;112(4):731-739.e1. doi:10.1016/j. fertnstert.2019.05.018

57. Goldman KN, Chenette D, Arju R, et al. mTORC1/2 inhibition preserves ovarian function and fertility during genotoxic chemotherapy. PNAS. 2017;114(12):3186-3191. doi:10.1073/ pnas. 1617233114

58. Codacci-Pisanelli G, Del Pup L, Del Grande M, Peccatori FA. Mechanisms of chemotherapy-induced ovarian damage in breast cancer patients. Crit Rev Oncol Hematol. 2017;113:90-96. doi:10.1016/j.critrevonc.2017.03.009

59. Zhao J, Liu J, Chen K, et al. What lies behind chemotherapy-induced amenorrhea for breast cancer patients: a meta-analysis. Breast Cancer Res Treat. 2014;145(1):113-128. doi:10.1007/s10549-014-2914-x

60. Lambertini M, Del Mastro L, Pescio MC, et al. Cancer and fertility preservation: international recommendations from an expert meeting. BMC Med. 2016;14(1):1. doi:10.1186/s12916-015-0545-7

61. Lambertini M, Campbell C, Bines J, et al. Adjuvant Anti-HER2 Therapy, Treatment-Related Amenorrhea, and Survival in Premenopausal HER2-Positive Early Breast Cancer Patients. JNCI J Natl Cancer Inst. 2019;111(1):86-94. doi:10.1093/jnci/djy094

62. Ejlertsen B, Tuxen MK, Jakobsen EH, et al. Adjuvant Cyclophosphamide and Docetaxel With or Without Epirubicin for Early TOP2A - Normal Breast Cancer: DBCG 07-READ, an OpenLabel, Phase III, Randomized Trial. J Clin Oncol. 2017;35 (23):2639-2646. doi:10.1200/JCO.2017.72.3494

63. Lambertini M, Ceppi M, Cognetti F, et al. Dose-dense adjuvant chemotherapy in premenopausal breast cancer patients: a pooled analysis of the MIG1 and GIM2 Phase III studies. Eur J Cancer. 2017;71:34-42. doi:10.1016/j.ejca.2016.10.030

64. Morarji K, McArdle O, Hui K, et al. Ovarian function after chemotherapy in young breast cancer survivors. Curr Oncol. 2017;24 (6):494. doi: $10.3747 / \mathrm{co} .24 .3335$

65. Abusief ME, Missmer SA, Ginsburg ES, Weeks JC, Partridge AH. The effects of paclitaxel, dose density, and trastuzumab on treatment-related amenorrhea in premenopausal women with breast cancer. Cancer. 2010;116(4):791-798. doi:10.1002/ cncr.24835
66. Tolaney SM, Barry WT, Dang CT, et al. Adjuvant Paclitaxel and Trastuzumab for Node-Negative, HER2-Positive Breast Cancer. $N$ Engl J Med. 2015;372(2):134-141. doi:10.1056/NEJMoa1406281

67. Ruddy KJ, Guo H, Barry W, et al. Chemotherapy-related amenorrhea after adjuvant paclitaxel-trastuzumab (APT trial). Breast Cancer Res Treat. 2015;151(3):589-596. doi:10.1007/s10549-015-3426-z

68. Gianni L, Pienkowski T, Im Y-H, et al. 5-year analysis of neoadjuvant pertuzumab and trastuzumab in patients with locally advanced, inflammatory, or early-stage HER2-positive breast cancer (NeoSphere): a multicentre, open-label, Phase 2 randomised trial. Lancet Oncol. 2016;17(6):791-800. doi:10.1016/S1470-2045(16)00163-7

69. Piccart M, Procter M, Fumagalli D, et al. Adjuvant Pertuzumab and Trastuzumab in Early HER2-Positive Breast Cancer in the APHINITY Trial: 6 Years' Follow-Up. J Clin Oncol. 2021: JCO.20.01204. doi:10.1200/JCO.20.01204

70. Deeks ED. Neratinib: first Global Approval. Drugs. 2017;77 (15):1695-1704. doi:10.1007/s40265-017-0811-4

71. Martin M, Holmes FA, Ejlertsen B, et al. Neratinib after trastuzumab-based adjuvant therapy in HER2-positive breast cancer (ExteNET): 5-year analysis of a randomised, double-blind, placebo-controlled, phase 3 trial. Lancet Oncol. 2017;18 (12):1688-1700. doi:10.1016/S1470-2045(17)30717-9

72. Molinelli C, Parisi F, Razeti MG, et al. Trastuzumab emtansine (T-DM1) as adjuvant treatment of HER2-positive early breast cancer: safety and efficacy. Expert Rev Anticancer Ther. 2020:1-10. doi:10.1080/14737140.2021.1857243

73. von Minckwitz G, Huang C-S, Mano MS, et al. Trastuzumab Emtansine for Residual Invasive HER2-Positive Breast Cancer. $N$ Engl J Med. 2019;380(7):617-628. doi:10.1056/NEJMoa1814017

74. Ruddy KJ, Trippa L, Hu J, et al. Abstract P2-13-02: chemotherapy-related amenorrhea (CRA) after adjuvant trastuzumab emtansine (T-DM1) compared to paclitaxel in combination with trastuzumab $(\mathrm{TH})$ (TBCRC033: ATEMPT trial). In: poster Session Abstracts. Am Assoc Cancer Rese. 2020:P2-13-02-P2-13-02. doi:10.1158/1538-7445.SABCS19-P2-13-02

75. Johnston SRD, Harbeck N, Hegg R, et al. Abemaciclib Combined With Endocrine Therapy for the Adjuvant Treatment of HR+, HER2 -, Node-Positive, High-Risk, Early Breast Cancer (monarchE). J Clin Oncol. 2020;38(34):3987-3998. doi:10.1200/JCO.20.02514

76. Mayer EL, Dueck AC, Martin M, et al. Palbociclib with adjuvant endocrine therapy in early breast cancer (PALLAS): interim analysis of a multicentre, open-label, randomised, phase 3 study. Lancet Oncol. 2021;22(2):212-222. doi:10.1016/S1470-2045(20)30642-2

77. Poggio F, Bruzzone M, Ceppi M, et al. Single-agent PARP inhibitors for the treatment of patients with BRCA-mutated HER2-negative metastatic breast cancer: a systematic review and meta-analysis. ESMO Open. 2018;3(4):e000361. doi:10.1136/esmoopen-2018000361

78. Winship AL, Griffiths M, Lliberos Requesens C, Sarma U, Phillips K-A, Hutt KJ. The PARP inhibitor, olaparib, depletes the ovarian reserve in mice: implications for fertility preservation. Hum Reprod. 2020;35(8):1864-1874. doi:10.1093/humrep/deaa128

79. Lambertini M, Preusser M, Zielinski CC. New emerging targets in cancer immunotherapy beyond CTLA-4, PD-1 and PD-L1: introducing an "ESMO Open - cancer Horizons" Series. ESMO Open. 2019;4:e000501. doi:10.1136/esmoopen-2019-000501

80. Robert C. A decade of immune-checkpoint inhibitors in cancer therapy. Nat Commun. 2020;11(1):3801. doi:10.1038/s41467-02017670-y

81. Duma N, Lambertini M. It is time to talk about fertility and immunotherapy. The Oncologist. 2020;25(4):277-278. doi:10.1634/ theoncologist.2019-0837

82. Postow MA, Sidlow R, Hellmann MD. Immune-related adverse events associated with immune checkpoint blockade. $N$ Engl J Med. 2018;378(2):158-168. doi:10.1056/NEJMra1703481 
83. Thompson JA. New NCCN Guidelines: recognition and Management of Immunotherapy-Related Toxicity. J Natl Compr Canc Netw. 2018;16(5S):594-596. doi:10.6004/jnccn.2018.0047

84. Bernhard J, Zahrieh D, Castiglione-Gertsch M, et al. Adjuvant Chemotherapy Followed By Goserelin Compared With Either Modality Alone: the Impact on Amenorrhea, Hot Flashes, and Quality of Life in Premenopausal Patients-The International Breast Cancer Study Group Trial VIII. J Clin Oncol. 2007;25 (3):263-270. doi:10.1200/JCO.2005.04.5393

85. Sinha N, Letourneau JM, Wald K, et al. Antral follicle count recovery in women with menses after treatment with and without gonadotropin-releasing hormone agonist use during chemotherapy for breast cancer. J Assist Reprod Genet. 2018;35(10):1861-1868. doi:10.1007/s10815-018-1203-8

86. Lambertini M, Boni L, Michelotti A, et al. Ovarian suppression with triptorelin during adjuvant breast cancer chemotherapy and long-term ovarian function, pregnancies, and disease-free survival: a randomized clinical trial. JAMA. 2015;314(24):2632. doi:10.1001/ jama.2015.17291

87. Leonard RCF, Adamson DJA, Bertelli G, et al. GnRH agonist for protection against ovarian toxicity during chemotherapy for early breast cancer: the Anglo Celtic Group OPTION trial. Ann Oncol. 2017;28(8):1811-1816. doi:10.1093/annonc/mdx184

88. Moore HCF, Unger JM, Phillips K-A, et al. Final Analysis of the Prevention of Early Menopause Study (POEMS)/SWOG Intergroup S0230. JNCI J Natl Cancer Inst. 2019;111(2):210-213. doi:10.1093/ jnci/djy 185
89. Lambertini M, Ceppi M, Poggio F, et al. Ovarian suppression using luteinizing hormone-releasing hormone agonists during chemotherapy to preserve ovarian function and fertility of breast cancer patients: a meta-analysis of randomized studies. Ann Oncol. 2015;26(12):2408-2419. doi:10.1093/annonc/mdv374

90. Rugo HS, Rosen MP. Reducing the long-term effects of chemotherapy in young women with early-stage breast cancer. JAMA. 2011;306 (3). doi:10.1001/jama.2011.1019

91. Zhang Y, Ji Y, Li J, et al. Sequential versus simultaneous use of chemotherapy and gonadotropin-releasing hormone agonist ( $\mathrm{GnRHa})$ among estrogen receptor (ER)-positive premenopausal breast cancer patients: effects on ovarian function, disease-free survival, and overall survival. Breast Cancer Res Treat. 2018;168(3):679-686. doi:10.1007/s10549-018-4660-y

92. Regan MM, Walley BA, Francis PA, et al. Concurrent and sequential initiation of ovarian function suppression with chemotherapy in premenopausal women with endocrine-responsive early breast cancer: an exploratory analysis of TEXT and SOFT. Ann Oncol. 2017;28 (9):2225-2232. doi:10.1093/annonc/mdx285

93. Dolmans -M-M, Taylor HS, Rodriguez-Wallberg KA, et al. Utility of gonadotropin-releasing hormone agonists for fertility preservation in women receiving chemotherapy: pros and cons. Fertil Steril. 2020;114(4):725-738. doi:10.1016/j.fertnstert.2020.08.011

94. Anderson RA, Clatot F, Demeestere I, et al. Cancer survivorship: reproductive health outcomes should be included in standard toxicity assessments. Eur J Cancer. 2021;144:310-316. doi:10.1016/j.ejca.2020.11.032

\section{Publish your work in this journal}

Breast Cancer - Targets and Therapy is an international, peer-reviewed open access journal focusing on breast cancer research, identification of therapeutic targets and the optimal use of preventative and integrated treatment interventions to achieve improved outcomes, enhanced survival and quality of life for the cancer patient.
The manuscript management system is completely online and includes a very quick and fair peer-review system, which is all easy to use. Visit http://www.dovepress.com/testimonials.php to read real quotes from published authors. 